\title{
Emergency physicians' practices and attitudes regarding procedural anaesthesia for nasogastric tube insertion
}

\author{
G A Juhl, G P Conners
}

Emerg Med J 2005;22:243-245. doi: 10.1136/emj.2004.015602

\begin{abstract}
Objective: To determine practice and attitudes of emergency physicians regarding procedural anaesthesia for nasogastric tube insertion (NGT).

Methods: Survey of resident/attending emergency physicians working in a tertiary care medical centre.

Results: Of 68 physicians, 46 responded: $98 \%$ believed that awake and alert patients find NGT insertion uncomfortable/ painful; 93\% used measures to reduce this, most commonly lubricant gel, topical anaesthetic spray, lidocaine gel, and distraction/use of a child life worker; $28 \%$ believed these provided adequate pain control and $37 \%$ believed they were inadequate. Topical anaesthetic spray, lidocaine gel, and nebulised/atomised anaesthetics were believed the most practical to administer and $44 \%$ actually used these. Nebulised/atomised anaesthetics, systemic anxiolytics, and topical anaesthetic spray were believed the most effective at pain control but only $24 \%$ actually used these. While $39 \%$ of respondents were satisfied with their current practice, $46 \%$ were dissatisfied: $91 \%$ would change their practice if new literature were to show a convenient way to effectively reduce this pain.

Conclusions: Emergency physicians do not actually use the measures they believe are most practical/most effective at reducing the pain associated with NGT insertion. Thus, there may be a barrier to the use of these measures. Improvement in procedural anaesthesia for NGT insertion in emergency departments is needed and desired by emergency physicians.
\end{abstract}

lisis nsertion of a nasogastric tube is the most painful of all routine emergency department procedures. ${ }^{1}$ There is a large, growing body of literature describing techniques for reducing this discomfort. Several topical, sprayed, atomised, and nebulised drugs, alone and in combination, have proved safe and successful. ${ }^{2-7}$ Despite the variety of available effective agents for reducing the pain of nasogastric tube insertion, anaesthesia is often not provided to patients undergoing this procedure. ${ }^{1}$ To our knowledge, there are no data addressing physicians' attitudes and practices regarding the provision of procedural anaesthesia for insertion of nasogastric tubes. Understanding these aspects, however, may provide important information when designing efforts promoting use of effective pain control measures during nasogastric tube insertion. ${ }^{8}$ The purpose of our study was to determine practice and attitudes of emergency physicians regarding procedural anaesthesia for nasogastric tube insertion.

\section{METHODS}

We distributed an anonymous survey to all resident and attending (faculty) emergency physicians who primarily work in the emergency department of a large, urban, US academic tertiary care medical centre with a fully accredited three year emergency medicine residency programme. The survey (see box) was designed by us and piloted with another physician group prior to administration. Survey questions concerned belief that nasogastric tube insertion causes pain, measures reducing discomfort, and satisfaction with these procedures. The discomfort reducing measures listed included some commonly used measures that are not anaesthetics, such as use of lubrication, anxiolytics, vasoconstrictors, and distraction or use of a child life worker (a non-physician health professional who works with children and families to reduce the stress associated with medical experiences and procedures). Responses were transferred without identifiers into a computerised database for analysis. The study protocol was reviewed and approved as exempted by the University of Rochester Research Subjects Review Board.

\section{RESULTS}

The survey was distributed to 38 emergency medicine resident physicians and 30 attending physicians; 46/68 (68\%) were completed and returned, including 28 by resident physicians $(74 \%)$ and 18 by attending physicians (60\%). Of the respondents, 98\% believed (agreed or strongly agreed) that awake and alert patients find nasogastric tube insertion uncomfortable or painful; 93\% agreed or strongly agreed that they used measures to reduce this pain/discomfort; and $28 \%$ believed (agreed or strongly agreed) that these measures are adequate for control of the pain/discomfort of nasogastric tube insertion, $37 \%$ believed (disagreed or strongly disagreed) that these measures are inadequate, and 35\% were neutral. Only 39\% of respondents were satisfied with their current practice (agreed or strongly agreed), 46\% were dissatisfied (disagreed or strongly disagreed), and $91 \%$ said they would change their practice if new literature were to show a convenient way to effectively reduce pain/discomfort.

Of pain control measures actually used by respondents, lubricant gel $(78 \%)$, topical anaesthetic spray (65\%), lidocaine gel $(54 \%)$, and distraction or use of a child life worker (17\%) were most commonly mentioned (multiple responses were allowed). Of these, topical anaesthetic spray (35\%), lubricant gel (33\%) and lidocaine gel $(20 \%)$ were believed to provide patients the most comfort. Respondents believed that topical anaesthetic spray (26\%), lidocaine gel $(26 \%)$, and nebulised or atomised anaesthetics $(24 \%)$ were the most practical to administer, assuming they were readily available. The anaesthetics that the respondents believed were the most effective for nasogastric tube insertion, assuming they were readily available, were: nebulised or atomised anaesthetics $(26 \%)$, systemic anxiolytics $(20 \%)$, and topical anaesthetic spray (17\%). Only $44 \%$ of respondents actually used the measure they believed was the most practical for pain reduction. Only $24 \%$ actually used the measure they believed was the most effective at pain reduction. 


\section{Anonymous data collection survey}

Circle the most appropriate answers

(1) I am an:

- E M resident

- E M attending

(2) I believe that patients who are awake and alert find it uncomfortable or painful to have an NGT inserted:

- strongly agree

- agree

- neutral

- disagree

- strongly disagree

(3) I use measures to reduce patients' pain/discomfort while they are having NGTs inserted:

- strongly agree

- agree

- neutral

- disagree

- strongly disagree

(4) Before I insert an NGT, I routinely premedicate with or use (circle all that apply):

- Nothing

- Lubricant/jelly (e.g. Surgilube)

- Lidocaine jelly

- Vasoconstrictors (e.g. phenylephrine topical)

- Systemic anxiolytics (e.g. diazepam IV)

- Systemic analgesics (e.g. morphine IV)

- Topical spray anesthetic (e.g. Cetacaine)

- Atomized anesthetic (e.g. lidocaine)

- Nebulized anesthetic (e.g. lidocaine)

- Child life worker/distraction

- Other

(5) Which one of the above measures (in \#4) that you routinely use, provides the most comfort for the patient?

(6) The above measure (in \#5) is adequate treatment for the pain/discomfort of NGT insertion:

- strongly agree

- agree

- neutral

- disagree

- strongly disagree

(7) Which premedication/intervention (if readily available) would be most PRACTICAL to reduce discomfort during NGT insertion in the ED (circle one):

- Nothing

- Lubricant/jelly (e.g. Surgilube)

- Lidocaine jelly

- Vasoconstrictors (e.g. phenylephrine topical)

- Systemic anxiolytics (e.g. diazepam IV)

- Systemic analgesics (e.g. morphine IV)

- Topical spray anesthetic (e.g. Cetacaine)
- Atomized anesthetic (e.g. lidocaine)

- Nebulized anesthetic (e.g. lidocaine)

- Child life worker/distraction

- Other

(8) Which premedication/intervention (if readily available) would be most EFFECTIVE in reducing discomfort during NGT insertion in the ED (circle one):

- Nothing

- Lubricant/jelly (e.g. Surgilube)

- Lidocaine jelly

- Vasoconstrictors (e.g. phenylephrine topical)

- Systemic anxiolytics (e.g. diazepam IV)

- Systemic analgesics (e.g. morphine IV)

- Topical spray anesthetic (e.g. Cetacaine)

- Atomized anesthetic (e.g. lidocaine)

- Nebulized anesthetic (e.g. lidocaine)

- Child life worker/distraction

- Other

(9) I am satisfied with my current practice of relieving pain/discomfort with NGT insertion:

- strongly agree

- agree

- neutral

- disagree

- strongly disagree

(10) I would change the way I place NGTs if new literature were to show a convenient way to effectively reduce patient pain/discomfort during NGT placement:

- strongly agree

- agree

- neutral

- disagree

- strongly disagree

\section{DISCUSSION}

Under-treatment of patient pain and discomfort has been recognised as a significant problem in emergency medicine. ${ }^{8-10}$ Nearly all the emergency physicians responding to our survey were aware that patients who are awake and alert find nasogastric tube insertion painful or uncomfortable. Although $93 \%$ of our respondents took measures to reduce this pain, only $28 \%$ believed that these measures provide adequate pain control.

Measures actually taken to reduce the pain of nasogastric tube insertion varied widely, with lubricant gel, topical anaesthetic spray, and lidocaine gel each used by over half of the respondents; these were also the three measures, of those actually used, that were believed most effective at providing comfort. Of measures that are available, however, those that respondents believed were the most effective are nebulised or atomised anaesthetics, systemic anxiolytics, and topical anaesthetic spray. Available measures they believed are the most practical were topical anaesthetic spray, lidocaine gel, and nebulised or atomised anaesthetics. Only topical anaesthetic spray appeared on all the lists, suggesting a barrier to use of the other effective or practical measures. This is further suggested by the finding that less than half of our respondents actually used the measure they believed is the most practical for pain reduction, and less than a quarter 
actually used the measure they believed is the most effective at pain reduction. It is therefore not surprising that nearly half of our respondents were dissatisfied with their current measures to reduce the discomfort of nasogastric tube insertion, and that $91 \%$ were willing to change their practice should the literature show a better method.

The effectiveness of anaesthetics to reduce the pain of nasogastric tube insertion has been well proven. Various topical anaesthetics with vasoconstrictors, especially nebulised, atomised, or gel lidocaine, have proved effective at reducing the pain of nasogastric tube insertion. ${ }^{2-6}$ Although commonly used by our respondents, lubricant gel alone has been shown to poorly reduce this pain. ${ }^{4}$ Although we did not specifically enquire in our survey as to the nature of the barriers to physicians' use of more effective anaesthetics, those suggested by other authors, including physicians' under-appreciation of pain, concerns about side effects, limited availability of desired medications, inadequate training in pain relief, and administrative barriers to anaesthetic use are likely contributors. ${ }^{811} 12$

In summary, we present evidence that the measures actually used to provide pain control for nasogastric tube insertion in the emergency department differ from those believed to be the most practical and the most effective. Many of the emergency physicians we surveyed were dissatisfied with this aspect of their practice, and the large majority was willing to change. Improvement in procedural anaesthesia for nasogastric tube insertion in the emergency department is needed and desired by emergency physicians.

\section{CONTRIBUTORS}

Dr Juhl initiated the study design, collected the data, analysed a part of the data, and did part of the writing. Dr Conners finalised the study design, analysed part of the data, and finalised the writing; he acts as guarantor for this paper.

\section{Authors' affiliations}

G A Juhl, G P Conners, Department of Emergency Medicine, University of Rochester School of Medicine and Dentistry, Rochester, NY, USA

Competing interests: none declared

Correspondence to: Dr G P Conners, Department of Emergency Medicine, 601 Elmwood Avenue, Box 655, Rochester, NY 14642, USA; gregory_conners@urmc.rochester.edu

Accepted for publication 3 May 2004

\section{REFERENCES}

1 Singer AJ, Richman PB, Kowalska A, et al. Comparison of patient and practitioner assessments of pain from commonly performed emergency department procedures. Ann Emerg Med 1999;33:652-8.

2 Ducharme J, Matheson K. What is the best topical anesthetic for nasogastric insertion? A comparison of lidocaine gel, lidocaine spray, and atomized cocaine. J Emerg Nurs 2003;29:427-30.

3 West HH. Topical anesthesia for nasogastric tube placement. Ann Emerg Med 1982;11:645.

4 Singer JK, Konia N. Comparison of topical anesthetics and vasoconstrictors vs. lubricants prior to nasogastric intubation: a randomized, controlled trial. Acad Emerg Med 1999:6:184-90.

5 Spektor M, Kelley J, Kelley J, et al. Nebulized or sprayed lidocaine as anesthesia for nasogastric intubations. Acad Emerg Med 2000;7:406-8.

6 Wolfe TR, Fosnocht DE, Linscott MS. Atomized lidocaine as topical anesthesia for nasogastric tube placement: a randomized, double-blind, placebocontrolled trial. Ann Emerg Med 2000;35:421-5.

7 Nott MR, Hughes JH. Topical anaesthesia for the insertion of nasogastric tubes. Eur J Anaesthesiol 1995; 12:287-90.

8 Maglinte DDT, Cordell WH. Strategies for reducing the pain and discomfort of nasogastric intubation. Acad Emerg Med 1999;6:166-9.

9 Afilalo M, Cantees K, Ducharme J. Current pain-control practices and research. Ann Emerg Med 1996;27:404-7.

10 Ducharme J. Proceedings from the First International Symposium on Pain Research in Emergency Medicine: Foreward. Ann Emerg Med 1996;27:399-403.

11 Middleton C. Barriers to the provision of effective pain management. Nurs Times 2004; 100:42-5.

12 Huang N, Cunningham F, Laurito $C$, et al. Can we do better with postoperative pain management? Am J Surg 2001;182:440-8. 